\title{
DETECTION OF VANCOMYCIN RESISTANT ENTEROCOCCI (VRE) IN HOSPITALIZED PATIENTS AND COMPARISION OF KIRBY-BAUER DISC DIFFUSION AND VANCOMYCIN SCREEN AGAR METHOD.
}

\author{
K. Vidyasagar, Nandan. T. M, R. Ravikumar
}

1. Senior Resident, Department of Neuromicrobiology, National Institute of Mental Health \& Neuro Sciences (NIMHANS), Bangalore.

2. Senior Resident, Department of Neuromicrobiology, National Institute of Mental Health \& Neuro Sciences (NIMHANS), Bangalore.

3. Professor \& Head, Department of Neuromicrobiology, National Institute of Mental Health \& Neuro Sciences (NIMHANS), Bangalore.

\section{CORRESPONDING AUTHOR}

Dr. R. Ravikumar, MD, Professor \& Head,

Department of Neuromicrobiology, NIMHANS, Hosur Road, Bangalore-560029

E-mail: ravikumarbly@yahoo.co.uk

Ph: +919448073965

ABSTRACT: The increasing occurrence of Enterococcus species, worldwide, since late 1980s, is of particular concern due to the emergence of Vancomycin Resistant Enterococci (VRE). The appearance of VRE has limited the therapeutic options available for clinicians. VRE infection is the most common type of infection acquired by patients while hospitalized. Patients at risk for VRE are those who are already ill, and hospitalized, including individuals with diabetes, elderly, ICU patients, kidney failure patients, or patients requiring catheters.

Present study was undertaken to detect vancomycin resistance in enterococcal isolates from hospitalized patients and the comparison of Kirby-Bauer disc diffusion method and vancomycin agar screen method to screen for vancomycin resistance.

A total of 45 enterococcal isolates from various samples of hospitalized patients were speciated by standard biochemical reactions and screened to detect vancomycin resistance by Kirby-Bauer disc diffusion method and vancomycin agar screen method. Out of 45 enterococcal isolates, 26 (57.77\%) were Enterococcus faecalis, 18 (40\%) were Enrerococcus faecium and $1(3.33 \%)$ was Enterococcus avium. Resistance to vancomycin was $29 \%$ (13 isolates) where as $71 \%$ (32 isolates) were sensitive. By Kirby-Bauer disc diffusion method, 1 isolate (2\%) showed resistance to vancomycin whereas by vancomycin screen agar, 13 (29\%) showed resistance.

The study highlights that vancomycin screen agar method is more sensitive than KirbyBauer disc diffusion method for detecting vancomycin resistance in enterococcal isolates. This study also signals the emergence of VRE in the hospital and highlights the importance of screening for VRE in enterococcal isolates from various samples.

KEY-WORDS: Vancomycin resistant Enterococci, Kirby-Bauer disc diffusion, vancomycin screen agar

INTRODUCTION: The increasing occurrence of Enterococcus species, worldwide, since late 1980s, is of particular concern due to the emergence of Vancomycin Resistant Enterococci (VRE). ${ }^{1}$ VRE has also been reported from some parts of India. ${ }^{2-3}$ The appearance of VRE has limited the therapeutic options available for clinicians.VRE infection is the most common type of infection acquired by patients while they are hospitalized. ${ }^{4}$ Patients at risk for VRE are those

Journal of Evolution of Medical and Dental Sciences/Volume1/Issue5/November-2012Page-882 
who are already ill, and hospitalized, including individuals with diabetes, elderly, ICU patients, kidney failure patients, or patients requiring catheters. 4

Present study was undertaken to detect Vancomycin resistance in enterococcal isolates from hospitalized patients and comparison of Kirby-Bauer disc diffusion method and Vancomycin agar screen method for Vancomycin resistance screening.

MATERIALS AND METHODS: A total of 45 enterococcal isolates, 28 from urine, 6 from brain abscess, 4 from CSF, 2 from blood, 2 from wound swab, 2 from ear swab, and 1 from tracheal secretion, were included in the study. Refer Table1 for details. They were identified and speciated by standard biochemical tests. ${ }^{5}$

Susceptibility to Vancomycin was performed by Kirby-Bauer Disc Diffusion Method (KBDDM) ${ }^{6}$ on Mueller Hinton Agar by using $30 \mu \mathrm{g}$ Vancomycin disc (HiMedia).

Vancomycin resistance was also determined by Vancomycin agar screen method using $6 \mu \mathrm{g} / \mathrm{ml}$ of Vancomycin incorporated in Brain Heart Infusion (BHI) agar. ${ }^{6}$ BHI agar with $6 \mu \mathrm{g}$ of Vancomycin was inoculated from a direct colony suspension equivalent to a 0.5 McFarland standard using a swab. The plates were incubated at $35 \pm 2{ }^{\circ} \mathrm{C}$ for 24 hours and later examined carefully for evidence of small colonies ( $>1$ colony) or a film of growth, indicating Vancomycin resistance. ${ }^{6}$

RESULTS: Out of the 45 enterococcal isolates, $57.77 \%$ (26) were Enterococcus faecalis, $40 \%$ (18) were Enterococcus faecium and 3.33\% (1) were Enterococcus avium. Refer Table2 for details.

$29 \%$ (13 isolates) were resistant to Vancomycin where as $71 \%$ (32 isolates) were sensitive. Refer Table3 for details.

One of the isolates (2\%) showed resistance to Vancomycin by Kirby-Bauer disc diffusion method (Figure 1). By Vancomycin screen agar, 13 isolates showed growth, giving an overall VRE positivity of $29 \%$ (Figure 2).

In this study, all the $13 \mathrm{VRE}$ isolates were Enterococcus faecium.

In this study, the Vancomycin screen agar method was found to be more sensitive in comparison to Kirby-Bauer disc diffusion method for detecting Vancomycin resistance in enterococcal isolates (Figure 3).

DISCUSSION: Vancomycin-resistant Enterococci (VRE) infection is the most common type of infection acquired by patients while hospitalized. ${ }^{4}$ Patients at risk for VRE are those who are already ill, and hospitalized, including individuals with diabetes, elderly, ICU patients, kidney failure patients, or patients requiring catheters. ${ }^{4}$

Enterococci are currently ascendant nosocomial pathogens, having become the second most common organisms recovered from nosocomial urinary tract and wound infections and the third most common cause of nosocomial bacteremia in the United States. ${ }^{7}$

There are the two types of Vancomycin resistance in Enterococci. The first type is intrinsic resistance. Isolates of Enterococcus gallinarum and E. casseliflavus/E. flavescens demonstrate an inherent, low-level resistance to Vancomycin. ${ }^{5}$

The second type of Vancomycin resistance in Enterococci is acquired resistance. Enterococci can become resistant to Vancomycin by acquisition of genetic information from another organism. Most commonly, this resistance is seen in E. faecium and E. faecalis, but also

Journal of Evolution of Medical and Dental Sciences/Volume1/Issue5/November-2012Page-883 
has been recognized in E. raffinosus, E. avium, E. durans, and several other Enterococcal species. ${ }^{5}$

Several genes, including vanA, vanB, vanC, vanD, and vanE, contribute to resistance to Vancomycin in Enterococci. ${ }^{5}$

Vancomycin resistance in enterococci has coincided with the increasing incidence of high-level enterococcal resistance to penicillin and amino glycosides, thus presenting a challenge for physicians who treat patients who have infections caused by these microorganisms ${ }^{8,9}$

Transmission of VRE by health care workers whose hands become transiently contaminated with the organism while caring for affected patients is probably the most common mode of nosocomial transmission. Transmission of VRE may also occur by way of contaminated medical equipments, although this is probably much less important than transmission by the hands of personnel. ${ }^{10}$

In this study, out of 45 enterococcal isolates, $57.77 \%$ (26) were Enterococcus faecalis, $40 \%$ (18) were Enterococcus faecium and $3.33 \%$ (1) were Enterococcus avium. This is in comparison with other Indian studies. ${ }^{2,3}$

De A.et al reported out of the 200 Enterococcus species, 55\% (110) were Enterococcus faecium, 31\% (62) were Enterococcus fecalis and 14\% (28) were other Enterococcus species. ${ }^{11}$

In this study, $29 \%$ (13 isolates) were resistance to Vancomycin where as $71 \%$ (32isolate) were sensitive. De A.et al reported $1.5 \%$ (3 isolates) were resistance to Vancomycin. ${ }^{11}$

In this study, one isolate (2\%) was showed resistance to Vancomycin by Kirby-Bauer disc diffusion method and by Vancomycin screen agar, 13 were showed growth, giving an overall VRE positivity of $29 \%$. De A.et al reported two isolates (1\%) were resistant to Vancomycin by Kirby-Bauer disc diffusion method and by Vancomycin agar screen method, three isolates showed growth, giving an overall VRE positivity of $1.5 \%$.

In this study, Enterococcus faecium was the commonest amongst VRE. De A.et al also reported Enterococcus faecium was the commonest amongst VRE.

This study showed Vancomycin screen agar method is more sensitive in comparison to Kirby-Bauer disc diffusion method for detecting Vancomycin resistance in enterococcal isolates

CONCLUSION: The study highlights that Vancomycin screen agar method is more sensitive than Kirby-Bauer disc diffusion method for detecting Vancomycin resistance in enterococcal isolates. This study also signals the emergence of VRE in the hospital and highlights the importance of screening for VRE in enterococcal isolates from various samples.

All laboratories should have effective detection methods for Vancomycin resistance, which will be helpful in reducing the morbidity and mortality due to VRE in hospitalized patients.

\section{REFERENCES:}

1. Zhanel GG, Laing NM, Nichol KA, Palatnick LP, Noreddin A, Hisanaga T, et al . Antibiotic activity against urinary tract infection (UTI) isolates of Vancomycin resistant Enterococci. J Antimicrob Chemother 2003; 52:382-88

2. Mathur P, Kapil A, Chandra R, Sharma P, Das B. Antimicrobial resistance in Enterococcus fecalis at a tertiary care center of Northern India. Indian J Med Res 2003; 118:25-28. 
3. Taneja N, Rani P, Emmanuel R, Sharma M. Significance of Vancomycin resistant enterococci from urinary specimens at a tertiary care center in Northern India. Indian J Med Res 2004;119:72-74.

4. Murray BE. Vancomycin-resistant enterococcal infections. N Eng J Med 2000; 342: 710-21.

5. Koneman's Color Atlas and Textbook of Diagnostic Microbiology. 5 th Ed. Lippincott, Philadelphia. 1997; pp577-650.

6. Performance Standards for Antimicrobial Susceptibility Testing. Seventeenth Informational Supplement. Clinical Laboratory Standards Institute. 2007; M100-S17;27(1):p53.

7. Schaberg, D. R., D. H. Culver, and R. P. Gaynes. 1991. Major trends in the microbial etiology of nosocomial infection. Am. J. Med. 91(Suppl. 3B):72S-75S.

8. 8.Handwerger S, Raucher B, Altarac D, et al. Nosocomial outbreak due to Enterococcus faecium highly resistant to Vancomycin, penicillin, and gentamicin. Clin Infect Dis 1993; 16:750-55.

9. CDC. Nosocomial enterococci resistant to Vancomycin -- United States, 1989-1993. MMWR 1993; 42:597-99.

10. Tornieporth, N. G., R. B. Roberts, J. John, A. Hafnier, and L. W. Riley. 1996. Risk factors associated with Vancomycin-resistant Enterococcus faecium infection or colonization in 145 matched case patients and control patients. Clin. Infect. Dis. 23:767-772.

11. De A, Bindlish A, Kumar S, Mathur M. Vancomycin resistant enterococci in a tertiary care hospital in Mumbai. Indian J Med Microbiol 2009; 27:375-76

Table 1: Enterococcal isolates from various specimens

\begin{tabular}{|c|c|c|}
\hline Sl no. & Specimen & Number \\
\hline 1 & Urine & 28 \\
\hline 2 & Brain abscess & 6 \\
\hline 3 & CSF & 4 \\
\hline 4 & Blood & 2 \\
\hline 5 & Wound swab & 2 \\
\hline 6 & Ear swab & 2 \\
\hline 7 & Tracheal secretions & 1 \\
\hline & Total & 45 \\
\hline
\end{tabular}

Table 2: Distribution of enterococcal isolates

\begin{tabular}{|c|c|c|c|}
\hline Sl.no & Isolate & Number of isolates & Percentage \\
\hline 1 & E. feacalis & 26 & $57.77 \%$ \\
\hline 2 & E. faecium & 18 & $40 \%$ \\
\hline 3 & E. avium & 1 & $2.22 \%$ \\
\hline & Total & 45 & 100 \\
\hline
\end{tabular}




\section{ORIGINAL ARTICLE}

Table 3: Vancomycin susceptibility pattern of enterococcal isolates

\begin{tabular}{|c|c|c|}
\hline \multirow{2}{*}{$\begin{array}{c}\text { Total } \\
\text { number of } \\
\text { isolates }\end{array}$} & \multicolumn{2}{|c|}{$\begin{array}{c}\text { Vancomycin } \\
\text { susceptibility }\end{array}$} \\
\cline { 2 - 3 } & $\mathrm{S}$ & $\mathrm{R}$ \\
\hline 45 & 32 & $\begin{array}{c}13 \\
(71 \%)\end{array}$ \\
\hline
\end{tabular}

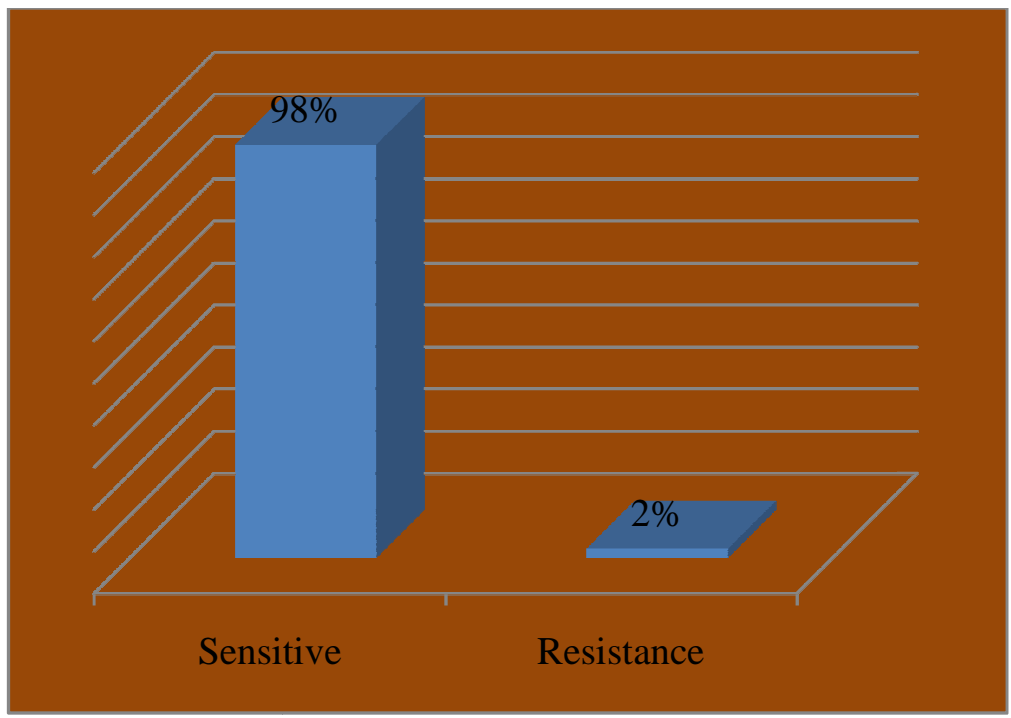

Figure1: Schematic representation of vancomycin susceptibility pattern of enterococcal isolates by disc diffusion method

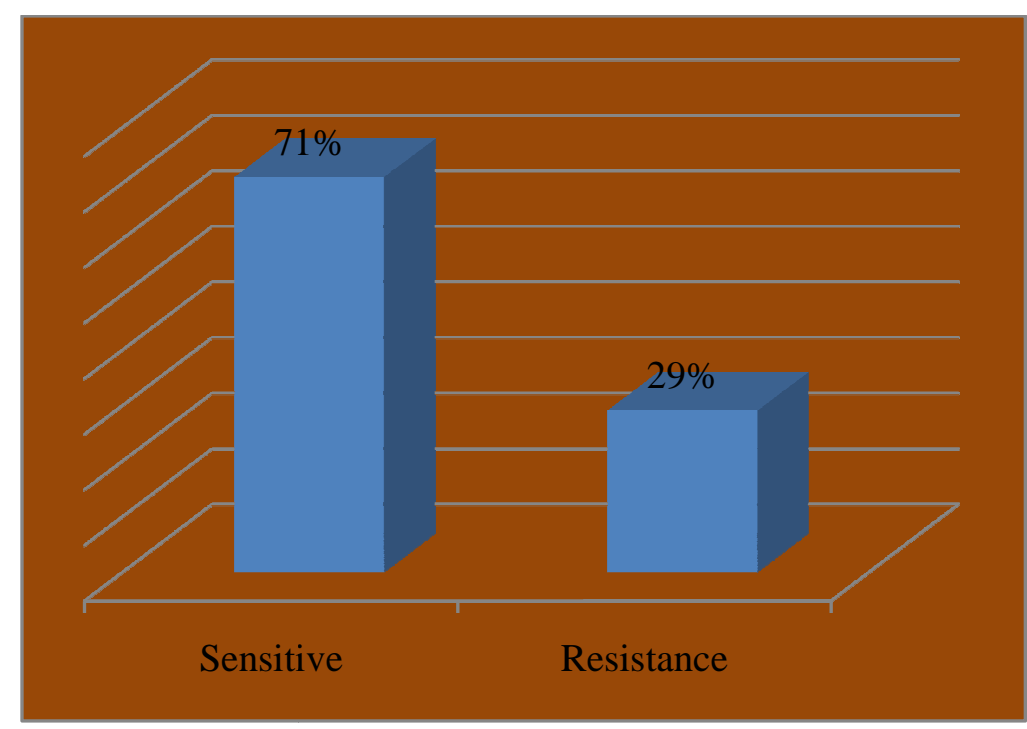

Figure2: Schematic representation of vancomycin susceptibility pattern of enterococcal isolates by vancomycin screen agar 


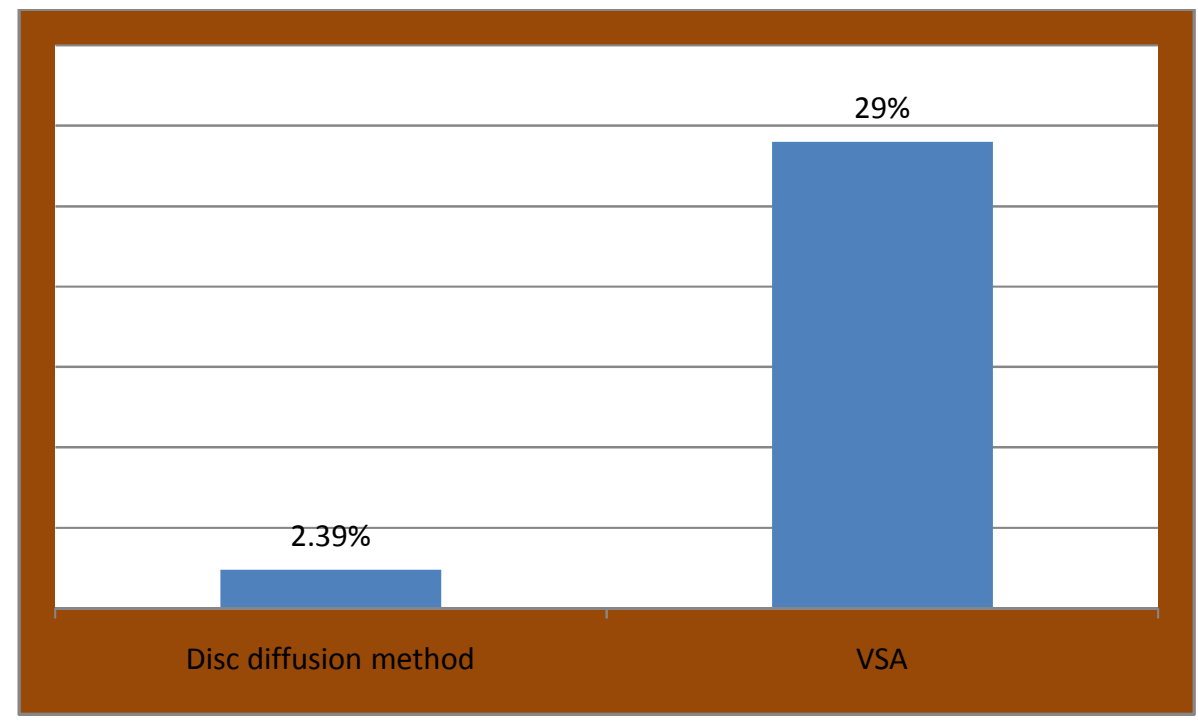

Figure 3: Comparison of enterococcal resistance by disc diffusion method and vancomycin screen agar 